\title{
Entropy, diffusivity and the energy landscape of a waterlike fluid
}

Cite as: J. Chem. Phys. 132, 234509 (2010); https://doi.org/10.1063/1.3429254

Submitted: 19 February 2010 . Accepted: 22 April 2010 . Published Online: 17 June 2010

Alan Barros de Oliveira, Evy Salcedo, Charusita Chakravarty, and Marcia C. Barbosa

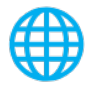

\section{ARTICLES YOU MAY BE INTERESTED IN}

Entropy, diffusivity, and structural order in liquids with waterlike anomalies

The Journal of Chemical Physics 125, 204501 (2006); https://doi.org/10.1063/1.2390710

Diffusivity, excess entropy, and the potential-energy landscape of monatomic liquids

The Journal of Chemical Physics 124, 014507 (2006); https://doi.org/10.1063/1.2140282

Excess entropy and crystallization in Stillinger-Weber and Lennard-Jones fluids

The Journal of Chemical Physics 143, 164512 (2015); https://doi.org/10.1063/1.4933420

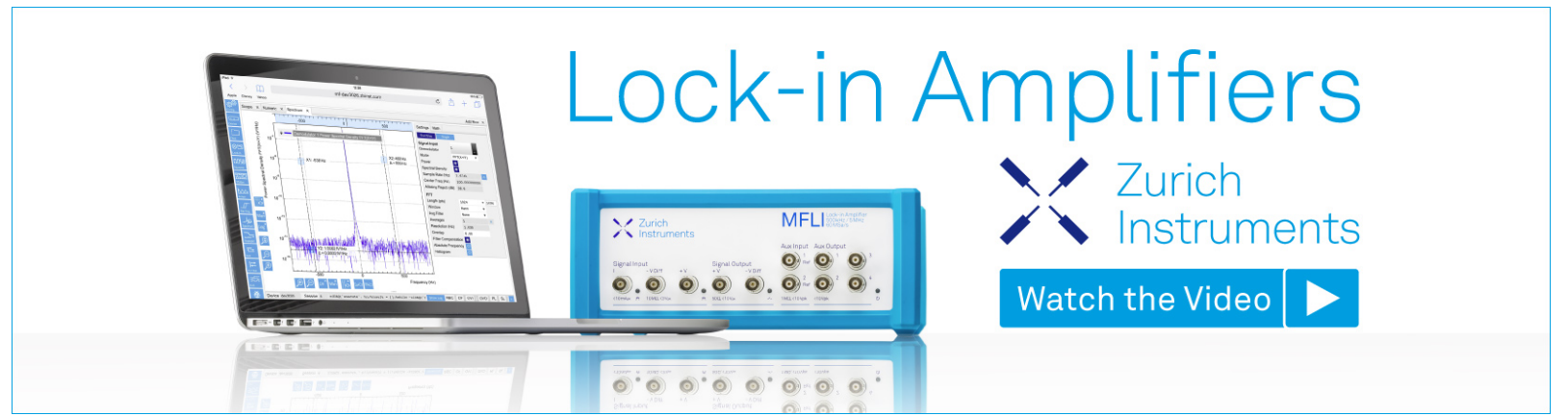

J. Chem. Phys. 132, 234509 (2010); https://doi.org/10.1063/1.3429254 


\title{
Entropy, diffusivity and the energy landscape of a waterlike fluid
}

\author{
Alan Barros de Oliveira, ${ }^{1, a}$ Evy Salcedo, ${ }^{2, b)}$ Charusita Chakravarty, ${ }^{3, c)}$ and \\ Marcia C. Barbosa ${ }^{4, d)}$ \\ ${ }_{1}^{1}$ Departamento de Física, Universidade Federal de Ouro Preto, Ouro Preto, MG 35400-000, Brazil \\ ${ }^{2}$ Departamento de Física, Universidade Federal de Santa Catarina, Florianópolis, SC 88010-970, Brazil \\ ${ }^{3}$ Department of Chemistry, Indian Institute of Technology-Delhi, New Delhi, 110016, India \\ ${ }^{4}$ Instituto de Física, Universidade Federal do Rio Grande do Sul, Porto Alegre, RS 91501-970, Brazil
}

(Received 19 February 2010; accepted 22 April 2010; published online 17 June 2010)

\begin{abstract}
Molecular dynamics simulations and instantaneous normal mode (INM) analysis of a fluid with core-softened pair interactions and waterlike liquid-state anomalies are performed to obtain an understanding of the relationship between thermodynamics, transport properties, and the potential energy landscape. Rosenfeld scaling of diffusivities with the thermodynamic excess and pair correlation entropy is demonstrated for this model. The INM spectra are shown to carry information about the dynamical consequences of the interplay between length scales characteristic of anomalous fluids, such as bimodality of the real and imaginary branches of the frequency distribution. The INM spectral information is used to partition the liquid entropy into two contributions associated with the real and imaginary frequency modes; only the entropy contribution from the imaginary branch captures the nonmonotonic behavior of the excess entropy and diffusivity in the anomalous regime of the fluid. (C) 2010 American Institute of Physics.
\end{abstract}

[doi:10.1063/1.3429254]

\section{INTRODUCTION}

The potential energy surface (PES), $U(\mathbf{r})$, is the configurational energy of a system of $N$ particles as a function of the $3 N$-dimensional position vector, r. Energy landscape approaches focus on the connections between crucial topographical features of the PES and the thermodynamic and kinetic properties of liquids. ${ }^{1-3}$ Structure and dynamics in simple liquids is dominated by strong, short-range, repulsions with weak, long-range attractions. ${ }^{4}$ Since the hardsphere fluid with a single length scale is a very good zerothorder model for such systems, the relationship between the energy landscape, thermodynamics, and mobility is relatively simple in such systems. Energy landscape analysis of anomalous fluids, such as water and silica, suggests that key features of the PES surface are significantly different from PESs of simple liquids. ${ }^{5-9}$ Recent work demonstrates that softening the core-repulsions in liquids with isotropic, pair-additive interactions allows one to generate a range of anomalous behavior that mimics the behavior of structurally more complex fluids, ${ }^{10-24}$ suggesting that significant restructuring of the energy landscape can be induced by simple modifications of the pair interactions.

In this study, we explore the energy landscape of such a core-softened fluid to understand the microscopic origins of waterlike liquid state anomalies. Our analysis of the PES focuses on understanding the entropy scaling relationships that are very useful for connecting structure, mobility and entropy for a wide range of simple $\mathrm{e}^{25-31}$ and anomalous ${ }^{32-35}$

\footnotetext{
${ }^{a)}$ Electronic mail: oliveira@iceb.ufop.br.

${ }^{b}$ Electronic mail: esalcedo@fsc.ufsc.br.

${ }^{c}$ Electronic mail: charus@chemistry.iitd.ernet.in.

${ }^{\mathrm{d})}$ Electronic mail: marcia.barbosa@ufrgs.br.
}

liquids, confined fluids and polymeric melts. ${ }^{36}$ The excess entropy $\left(S_{\text {ex }}\right)$ measures the reduction in the entropy $(S)$ of a liquid relative to an ideal gas $\left(S_{\mathrm{id}}\right)$ at the same temperature and density due to structural correlations. The effect of fluid structure on the entropy can be formally expressed as

$$
S_{\mathrm{ex}}=S-S_{\mathrm{id}}=S_{2}+S_{3}+\ldots,
$$

where $S_{n}$ is the entropy contribution due to $n$-particle spatial correlations. ${ }^{37-41}$ The pair correlation contribution to the excess entropy per particle of a one-component fluid of structureless particles is given by

$$
s_{2}^{*}=-2 \pi \rho \int_{0}^{\infty}\{g(r) \ln g(r)-[g(r)-1]\} r^{2} d r,
$$

where $g(r)$ is the radial distribution function and $s_{2}^{*}$ $=S_{2} / N k_{B}$. The structural correlations which lower the entropy may intuitively be expected to reduce mobility by enhancing cage effects due to formation of shells of neighboring particles. This correlated decrease in entropy and mobility can be semiquantitatively captured through excess entropy scaling relations of the form

$$
X^{*}=A \exp \left(\alpha s_{\mathrm{ex}}^{*}\right),
$$

where $X^{*}$ are dimensionless transport properties with either macroscopic (Rosenfeld) or microscopic (Dzugutov) reduction parameters, $s_{\mathrm{ex}}^{*}$ is the excess entropy per particle in units of $k_{B}$. The Rosenfeld reduction parameters are given in units of the mean interparticle distance $d=(\rho)^{-1 / 3}$ and of the thermal velocity $v=\left(k_{B} T / m\right)^{-1 / 3}$ while the Dzygutov reduction parameter is calculated using the interparticle distance $\sigma$ and the collision frequency.

The scaling parameters, $\alpha$ and $A$, depend on the functional form of the underlying interactions. ${ }^{25-31}$ In the case of 
simple liquids, the excess entropy scaling parameters can be approximately set as $A \approx 0.6$ and $\alpha \approx 0.8$. In addition, for such fluids, the pair correlation entropy per particle, $s_{2}^{*}$, typically represents $85 \%-90 \%$ of the total excess entropy.

This paper focuses on the connection between the potential energy landscape (PEL) of a fluid and Rosenfeld excess entropy scaling of transport properties. Liquids in the stable, as opposed to the strongly supercooled regime, are characterized by a very high degree of connectivity between basins of local minima. This implies that the diffusivity, corresponding to the probability that a particle will make a successful move from its current position, will be proportional to the number of accessible configurational states, or $\exp \left(\alpha s_{\text {ex }}^{*}\right)$. In order to develop a quantitative test of this intuitive picture, the diffusivity and/or the entropy must be correlated with landscape-based quantities that are sensitive to basin connectivity. We also require an energy landscape approach that does not presume a time-scale separation between intra- and interbasin motions. An existing energy landscape approach that is simple to implement and satisfies these requirements is the instantaneous normal mode (INM) approach. $^{42-44}$ In the INM approach, the key quantity is the ensemble-averaged curvature distribution of the PES sampled by the system. For a system of $N$ particles, the mass-weighted Hessian associated with each instantaneous configuration is diagonalized to yield $3 N$ normal mode eigenvalues and eigenvectors and the ensemble-average of this distribution is referred to as the INM spectrum. The shorttime dynamics of the liquid can be derived from the INM spectra. Unlike in a crystalline solid, the INM spectrum of a liquid will have a substantial fraction of unstable modes with negative eigenvalues, corresponding to interbasin crossing modes or shoulder regions within the same inherent structure basin. The diffusivity is strongly correlated with the properties of the INM spectrum, specially the fraction of imaginary frequencies, in both simple liquids, such as Lennard-Jones and Morse, ${ }^{45-47}$ as well as molecular liquids, such as $\mathrm{CS}_{2}$ and $\mathrm{H}_{2} \mathrm{O}$. ${ }^{9,48,49}$ A refinement of the INM approach shows that interbasin crossing or double-well modes are critical for diffusional motion. ${ }^{50}$

Here we study the INM spectra of a liquid bound by isotropic, core-softened pair interactions which shows waterlike structural, density, entropy, and diffusional anomalies. The thermodynamic and transport properties of such a liquid is representative of structurally more complex anomalous liquids, including water ${ }^{19-21,51}$ and other tetrahedral liquids, such as $\mathrm{Te},{ }^{52} \mathrm{Ga}, \mathrm{Bi}^{53} \mathrm{~S},{ }^{54,55} \mathrm{Ge}_{15} \mathrm{Te}_{85},{ }^{56}$ silica, ${ }^{32,57-59}$ silicon, ${ }^{60}$ and $\mathrm{BeF}_{2}{ }^{32-34,57,61,62}$ Section II describes our coresoftened model fluid with isotropic interactions consisting of a sum of Lennard-Jones and Gaussian terms. The continuous nature of the pair interaction makes it very convenient for energy landscape analysis. The liquid state anomalies of this model, which have been described in detail elsewhere, ${ }^{14,15}$ are summarized. In Sec. III, we provide a summary of the relevant features of INM analysis. We also address the possibility of extracting thermodynamic quantities, including excess entropy, from INM spectra which has so far not been discussed in the literature. Section IV presents our results and Sec. V contains the conclusions.

\section{THE MODEL}

\section{A. PES}

We consider a three-dimensional (3D), core-softened fluid with isotropic pair interactions given by

$$
U(r)=4 \epsilon\left[\left(\frac{\sigma}{r}\right)^{12}-\left(\frac{\sigma}{r}\right)^{6}\right]+a \epsilon \exp \left[-\frac{1}{c^{2}}\left(\frac{r-r_{0}}{\sigma}\right)^{2}\right] \text {. }
$$

Equation (4) shows that the pair interaction is composed of a Lennard-Jones term, with characteristic energy and length scale parameters corresponding to $\epsilon$ and $\sigma$ respectively, plus a Gaussian well centered at a pair separation $r_{0}$ with depth $a \epsilon$ and width $c \sigma$. In this work, we use the parameters for Eq. (4) as $a=5, r_{0} / \sigma=0.7$, and $c=1$. This set of parameters generates a core-softened potential with a very small attractive minimum at $r \approx 3.8 \sigma$ and a soft, repulsive core lying between $\sigma$ and $3 \sigma$. All quantities in this paper are reported in reduced units with the $\sigma$ and $\epsilon$ as the reduced units of length and energy respectively. The waterlike structural, density, and diffusional anomalies of this model are briefly described in this section in order to provide a background to the INM results presented in Sec. IV.

\section{B. Molecular dynamics simulations}

Classical molecular dynamics (MD) simulations were used to study the model fluid described in the previous subsection. $N=500$ identical, structureless particles of mass $m$ were confined in a cubic box, of volume $V$, with periodic boundary conditions in all directions. All MD simulations were performed in the canonical $(N V T)$ ensemble with a time step of $0.002 \sigma \sqrt{m / \epsilon}$. A Nosé-Hoover thermostat with the coupling parameter equal to 2 was used to maintain the temperature. All simulations were initialized with the system in a face centered cubic configuration and further equilibrated over 250000 steps for each temperature, $T$, and density, $\rho$ $=N / V$. After the equilibration period was over, additional 500000 steps were used to sample the system. A cutoff radius $r_{c}=3.5 \sigma$ was employed for the potential Eq. (4). Diffusivities were computed using the Einstein relation. At each state point, 100 configurations were sampled and used to construct the INM spectra and associated quantities. We repeated the calculation for some state points using 500 configurations and found no significant difference.

\section{Density, diffusional, and structural anomalies}

Figure 1 illustrates the regions associated with the density, diffusional, and structural anomalies of the model fluid studied here in the density-temperature planes. The region of density anomaly corresponds to state points for which $(\partial \rho / \partial T)_{P}>0$ and is bounded by the locus of points for which the thermal expansion coefficient is zero. The translational diffusion coefficient as a function of $\rho^{*}=\rho \sigma^{3}$ goes as follows. For the low temperature isotherms, the diffusivity increases as the density is lowered, reaches a maximum at $\rho_{D \max }$ and decreases until it reaches a minimum at $\rho_{D \text { min. }}$. The locus of 


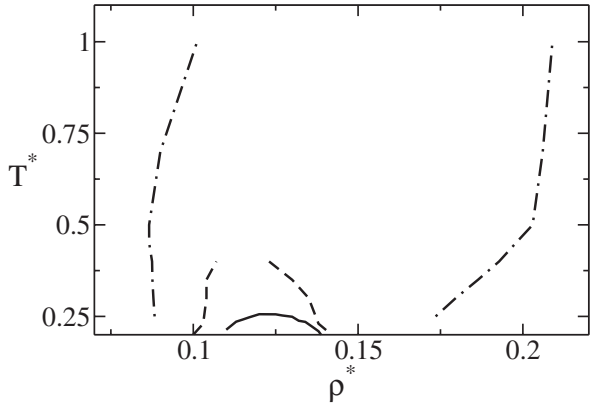

FIG. 1. Density vs temperature phase diagram for the model studied. The solid line limits the region of density anomaly, the dashed lines illustrate the region of diffusion anomaly and the dot-dashed lines show the region of structural anomaly.

extrema in the $D(\rho)$ curve mark the boundaries of the region of diffusional anomaly, as shown in Fig. 1 using dashed lines.

The region of structural anomaly of core-softened fluids is defined most simply using the translational or pair correlation order metric, defined as ${ }^{19}$

$$
t \equiv \int_{0}^{\xi_{c}}|g(\xi)-1| d \xi
$$

where $\xi \equiv r \rho^{1 / 3}$ is the interparticle separation scaled by the mean interparticle distance, $g(\xi)$ is the radial distribution function and $\xi_{c}$ is a scaled cutoff distance. In this work, we use $\xi_{c}=\rho^{1 / 3} L / 2$, where $L=V^{1 / 3}$. For a completely uncorrelated system (ideal gas) $g=1$ and $t$ vanishes. In a crystal, the presence of long-range translational $(g \neq 1)$ implies that $t$ depends on choice of $\xi_{c}$. In simple liquids, $t$ increases with isothermal compression. In liquids with waterlike anomalies at low temperatures, $t$ shows a nonmonotonic behavior. At a given temperature $T$, a structurally anomalous regime can be defined between densities $\rho_{t-\max }(T)$ and $\rho_{t-\min }(T)$ corresponding to locations of the maxima and minima in the translational order. In this structurally anomalous regime, shown using dot-dashed lines in Fig. 1, an increase in density induces a decrease in translational order. The nested structures of the anomalous regions is evident with the structurally anomalous regime enclosing the diffusion anomalous region which in turn encloses the region of density anomaly.

\section{INM ANALYSIS}

In this section, we define the INM spectrum and explore how it can be used to connect the energy landscape of a liquid with its thermodynamic entropy. The potential energy of configuration $\mathbf{r}$ near $\mathbf{r}_{0}$ can be written as a Taylor expansion of the form

$$
U(\mathbf{r})=U\left(\mathbf{r}_{0}\right)-\mathbf{F} \cdot \mathbf{z}+\frac{1}{2} \mathbf{r}^{\mathbf{T}} \cdot \mathbf{H} \cdot \mathbf{z}
$$

where $\mathbf{z}_{\mathbf{i}}=\sqrt{m_{i}}\left(\mathbf{r}_{\mathbf{i}}-\mathbf{r}_{0}\right)$ are the mass-scaled position coordinates of a particle $i$. The first and second derivatives of $U(\mathbf{r})$ with respect to the vector $\mathbf{z}$ are the force and the Hessian matrix, denoted by $\mathbf{F}$ and $\mathbf{H}$ respectively. The eigenvalues of the Hessian $\mathbf{H}$ are $\left(\left\{\omega_{i}^{2}\right\}, i=1,3 N\right)$ representing the squares of normal mode frequencies, and $\mathbf{W}(\mathbf{r})$ are the corresponding eigenvectors. In a stable solid, $\mathbf{r}_{\mathbf{0}}$ can be conveniently taken as the global minimum of the PES $U(R)$, which implies that $\mathbf{F}=0$ and $\mathbf{H}$ has only positive eigenvalues corresponding to oscillatory modes. The INM approach for liquids interprets $\mathbf{r}$ as the configuration at time $t$ relative to the configuration $\mathbf{r}_{0}$ at time $t_{0}$. Since typical configurations, $\mathbf{r}_{0}$ are extremely unlikely to be local minima, therefore $\mathbf{F} \neq 0$ and $\mathbf{H}$ will have negative eigenvalues. The negative eigenvalue modes are those which sample negative curvature regions of the PES, including barrier crossing modes. The ensemble-averaged INM spectrum, $\langle f(\omega)\rangle$, is defined as

$$
f(\omega)=\left\langle\frac{1}{3 N} \sum_{i=1}^{3 N} \delta\left(\omega-\omega_{i}\right)\right\rangle .
$$

Quantities that are convenient for characterizing the INM spectrum are (i) the fraction of imaginary frequencies, namely

$$
F_{\text {im }}=\int_{\text {im }} f(\omega) d \omega
$$

where the subscript in means that the integral is performed only in the imaginary branch; (ii) the fraction of real frequencies, that is

$$
F_{r}=\int_{r} f(\omega) d \omega,
$$

where the subscript $r$ indicates that the integral is performed only in the real branch and (ii) the mean square or Einstein frequency, $\omega_{E}$, given by

$$
\omega_{E}^{2}=\int \omega^{2} f(\omega) d \omega=\frac{\langle\operatorname{Tr} \mathbf{H}\rangle}{m(3 N-3)},
$$

where the last equality comes from using Eq. (7) and $\langle\operatorname{Tr} \mathbf{H}\rangle$ is the ensemble-averaged value of the trace of the Hessian.

The simplest approximation to the entropy that can be derived from the INM approach is to consider a liquid as a collection of $3 N$ simple harmonic oscillators vibrating at the Einstein frequency. The entropy of a one-dimensional harmonic oscillator with frequency $\omega$ is given by

$$
s_{\omega} / k_{B}=1-\ln (\beta \hbar \omega) .
$$

The entropy of an ideal gas of $\mathrm{N}$ particles in three dimensions will be given by

$$
\frac{S_{\mathrm{id}}}{N k_{B}}=\frac{5}{2}-\ln \left(\rho \Lambda^{3}\right),
$$

where $\Lambda=h / \sqrt{2 \pi m k_{B} T}$ is the thermal de Broglie wavelength. In three dimensions the entropy of the harmonic oscillators given by Eq. (11) is multiplied by 3 . The entropy per particle of the harmonic oscillator within Einstein approximations becomes $s_{\omega_{E}}$. In this case, the excess entropy of the Einstein model of the liquid is given by the subtraction of the ideal gas entropic contribution, Eq. (12), from $N s_{\omega_{E}} / k_{B}$ to give 


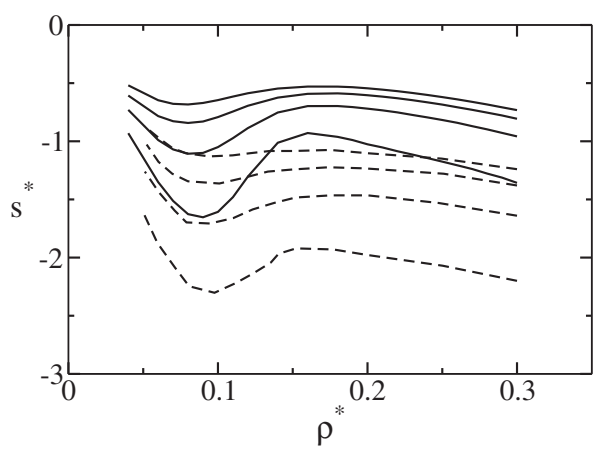

FIG. 2. The pair correlation entropy, $s_{2}^{*}$ (solid lines), and the excess entropy, $s_{\mathrm{ex}}^{*}$ (Ref. 35) (dashed lines) against density for fixed temperatures for $T^{*}=0.2,0.3,0.4,0.5$ from bottom to top.

$$
S_{\mathrm{ex}}^{\omega_{E}} / N k_{B}=\frac{1}{2}+\frac{3}{2} \ln \left(\frac{2 \pi k_{B} T \rho^{2 / 3}}{m \omega_{E}^{2}}\right),
$$

where $\omega_{E}$ is given by Eq. (10). This expression for the excess entropy forms the basis of quasiharmonic cell model approaches to understand entropy scaling of transport properties, which have had only limited success. ${ }^{27}$ In this study, we compare the Einstein frequency-based expression for the excess entropy with the pair correlation entropy to obtain a better microscopic insight into the differences.

An alternative approach is to consider the liquid to be composed on average of a set of $3 N F_{r}$ harmonic oscillators and a set of $3 N F_{\mathrm{im}}$ degrees of freedom associated with the imaginary or unstable modes. The total thermodynamic entropy of the liquid can be written as a sum of contributions from the real and imaginary branches,

$$
S=S_{r}+S_{\mathrm{im}} .
$$

Using Eq. (11), $S_{r}$ can be obtained by integrating the real branch of the INM distribution as follows:

$$
S_{r} / k_{B}=3 N \int_{r} f(\omega) s_{\omega}(\omega) d \omega,
$$

where $f(\omega)$ is the INM probability density at frequency $\omega$ given by Eq. (7). Contribution of the imaginary modes to the entropy must then be

$$
S_{\mathrm{im}}=S-S_{r}=S_{\mathrm{id}}+S_{\mathrm{ex}}-S_{r} .
$$

In the present work, we use the above equations to estimate $S_{r}$ and $S_{\text {im }}$; the per particle values of these quantities in units of $k_{B}$ are labeled $s_{r}^{*}$ and $s_{\text {im }}^{*}$. To our knowledge, this decomposition of the entropy has not been used previously. A somewhat parallel approach was used by Goddard et al. ${ }^{63}$ to treat the entropy of a liquid as a sum of contributions from a harmonic component and a hard-sphere fluid component.

\section{RESULTS}

\section{A. Excess entropy and diffusivity}

The excess entropy is defined as the difference between the entropy of the real fluid and that of the ideal gas at the same temperature and density. Figure 2 illustrates the density dependence of the excess (represented in the figure by dashed lines) and pair correlation entropy (represented in the
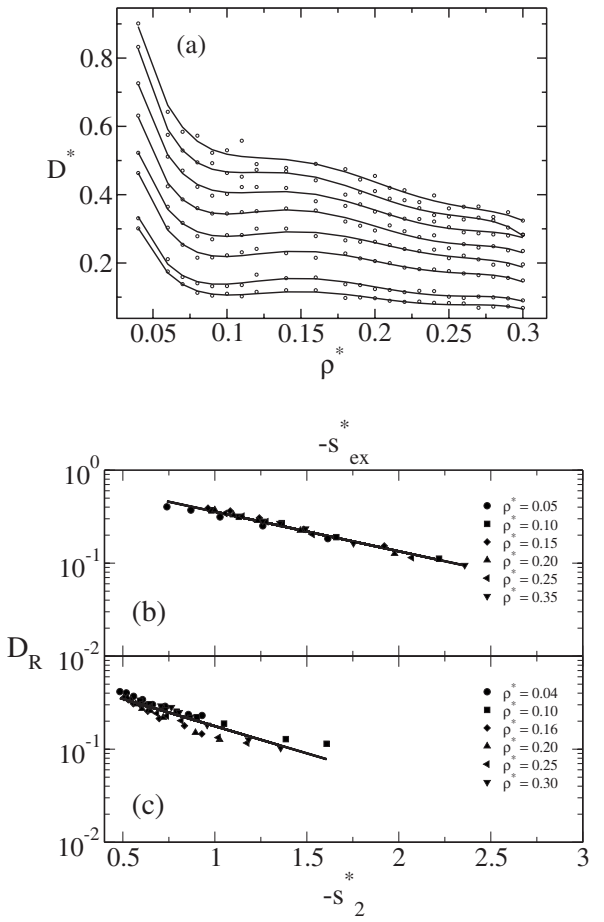

FIG. 3. (a) Diffusion vs reduced density for fixed temperatures $T^{*}$ $=0.2,0.23,0.30,0.35,0.40,0.45,0.50,0.55$ from bottom to top; (b) diffusion in Rosenfeld units vs the negative of the $-s_{\mathrm{ex}}^{*}$ and (c) diffusion in Rosenfeld units as a function of $-s_{2}^{*}$.

figure by solid lines) for four different isotherms. The values of the thermodynamic excess entropy, $s_{\mathrm{ex}}^{*}$, have been taken from the work of Mittal et al. ${ }^{35}$

The $s_{\mathrm{ex}}^{*}(\rho)$ curves at low temperatures show a pronounced excess entropy anomaly, corresponding to a rise in excess entropy on isothermal compression. Such an entropy anomaly is characteristic of waterlike liquids ${ }^{18,32-34}$ and contrasts with the behavior of simple liquids where free volume arguments are sufficient to justify a monotonic decrease in entropy on isothermal compression. Figure 2 also compares $s_{\mathrm{ex}}^{*}(\rho)$ and $s_{2}^{*}(\rho)$ curves at four temperatures. It is evident that $s_{2}^{*}$ essentially captures the anomalous behavior present in $s_{\mathrm{ex}}^{*}$. The effect of the higher-order multiparticle correlations terms in $s_{\mathrm{ex}}^{*}$ is to generate a downward shift in the values of the entropy and to attenuate the entropy anomaly. In the case of simple liquids, the residual multiparticle entropy, $\Delta s^{*}$ $=s_{\mathrm{ex}}^{*}-s_{2}^{*}$, is typically of the order of $10 \%-15 \%$ of $s_{\mathrm{ex}}^{*}$ for a fairly wide range of densities. Clearly in the case of the core-softened modeled fluids, the residual multiparticle entropy contribution is larger in magnitude and more strongly density dependent. The anomalous pair entropy regime at a given temperature is an interval of densities $\rho_{s 2_{\max }}<\rho$ $<\rho_{s 2_{\text {min }}}$ within which $\left(\partial S_{2} / \partial \rho\right)_{T}>0$. This can be identified from the locus of extrema in $s_{2}(\rho)$ shown in Fig. 10.

We now consider the scaling relationship between the diffusivity and the excess entropy. The diffusivity as a function of density for different isotherms is shown in Fig. 3(a). Clear maxima and minima in the $D(\rho)$ curves can be identified at low temperatures. Figures 3(b) and 3(c) show the scaling of the reduced diffusivity, $D_{R}$, with the excess, $s_{\mathrm{ex}}^{*}$, and pair, $s_{2}^{*}$, entropy. Using the Rosenfeld macroscopic re- 

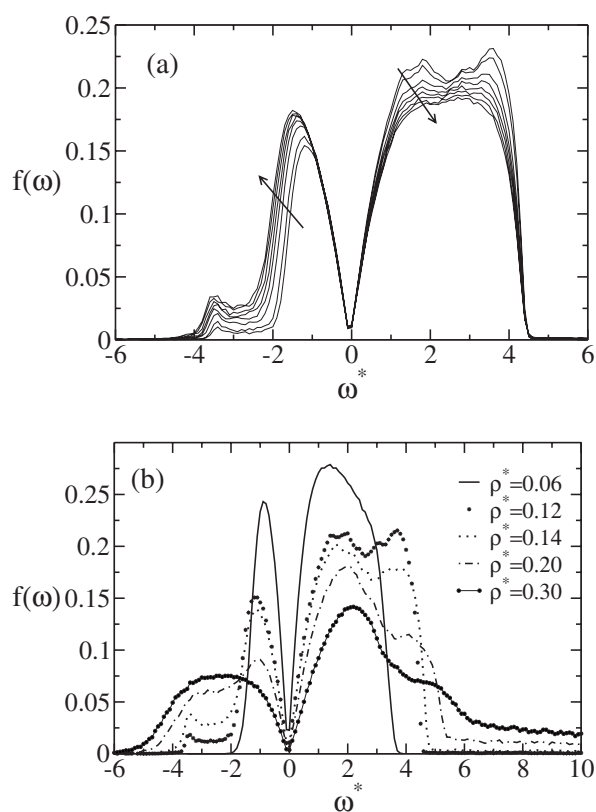

FIG. 4. (a) INM spectra as a function of frequency for $T^{*}=0.20,0.23,0.30$, $0.35,0.40,0.45,0.50$, and 0.55 and $\rho^{*}=0.11$. The arrows indicate the increase of the temperature. (b) INM spectra as a function of frequency for $T^{*}=0.20$ and $\rho^{*}=0.06,0.12,0.14,0.20,0.30$.

duction parameters for the length as $\rho^{-1 / 3}$ and the thermal velocity as $\left(k_{B} T / m\right)^{1 / 2}$, the dimensionless diffusivity is defined as

$$
D_{R} \equiv D \frac{\rho^{1 / 3}}{\left(k_{B} T / m\right)^{1 / 2}} .
$$

The scaling of the reduced diffusivity, $D_{R}$ with $s_{\mathrm{ex}}^{*}$ is excellent with $D_{R}=A e^{B s_{\text {ex }}^{*}}$ where $A=0.95$ and $B=0.98$. The scaling with the pair entropy, $s_{2}^{*}$, shows a weak isochore dependence and the line of best fit is obtained with $A=0.68$ and $B$ $=1.35$. The comparison between the Figs. 3(b) and 3(c) indicates that for our anomalous fluid, the diffusivity scaling with the pair correlation entropy is not as universal as with the excess entropy. This is likely to be a consequence of the presence of two density-dependent length scales, in such waterlike fluids when compared to simple liquids, such as the Lennard-Jones fluid.

\section{B. INM analysis}

Next, we present the results from our INM analysis of the simulation for the core-softened fluid. The INM spectra along the $\rho^{*}=0.11$ isochore for various temperatures is illustrated in Fig. 4(a) while the INM spectra along the $T^{*}$ $=0.20$ isotherm for various densities is illustrated in Fig. 4(b). The shape of the INM spectra have the characteristic real and imaginary branches. As in the case of Lennard-Jones and Morse liquids, ${ }^{45,58}$ the negative modes shrink in intensity and go to low frequencies as the temperature is decreased, while the peak of the real branch increases. Unlike in the case of simple liquids, however, both the real and the imaginary branch have a pronounced bimodality which can be clearly seen for the spectra along the $\rho^{*}=0.11$ isochore. which must be connected with two different length scales of the potential. Figure $4(\mathrm{~b})$ shows that along the $T^{*}=0.20$ iso-

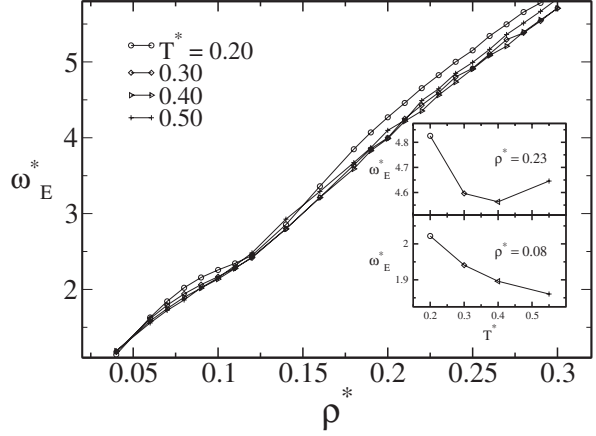

FIG. 5. Einstein frequency vs density for fixed temperature. The insets show the dependence with temperature for two fixed densities $\rho^{*}=0.08$ (bottom inset) and $\rho^{*}=0.23$ (top inset).

therm, the bimodality in the imaginary branch is most pronounced within the anomalous regime and is attenuated at both low and high densities. In contrast, the bimodality of the real branch persists even at high densities. It would be interesting to explore in future work if this bimodal frequency distribution results in multiple-time-scale behavior analogous to that seen in hydrogen-bonded liquids, such as water and methanol. ${ }^{51,64-66}$

The Einstein frequency is the second moment of the INM distribution, as defined in equation Eq. (10), and represents an effective frequency describing the very short-time, local dynamics of the particles. Figure 5 illustrates the behavior of Einstein frequency for the core-softened potential. For a fixed temperature, increasing density results in increase of $\omega_{E}^{*}=\omega \sqrt{m \sigma^{2} / \epsilon}$, indicating stronger trapping of the liquid particles in local cages. This is consistent with the behavior of simple liquids observed in earlier studies. ${ }^{45,58}$ The $\omega_{E}^{*}$ values is virtually independent of temperature for $\rho^{*} \approx 0.05$ and $\rho^{*} \approx 0.125$. At low densities, $\omega_{E}^{*}$ shows a small decrease with increasing temperature while at higher densities, there is a weak minimum in the $\omega_{E}^{*}$ at intermediate temperatures. The density dependence of $\omega_{E}^{*}$ carries no significant signatures of the diffusivity anomaly.

The fraction of imaginary modes, $F_{\text {im }}$, indicates how much the system samples regions with negative curvature which is known to be strongly correlated with the diffusivity. ${ }^{67}$ Figure 6 shows density dependence fraction of imaginary modes, $F_{\text {im }}$, for our core-softened anomalous fluid. In simple liquids, $F_{\text {im }}$ decreases with density and the graph $F_{\text {im }}$ versus $\rho^{*}$ always exhibit a negative slope. ${ }^{45,58}$ In contrast, for the core-softened potential studied here, $F_{\text {im }}$ shows very pronounced non-monotonic behavior For very low, $\rho<\rho_{F \text { min }}$, and very high, $\rho>\rho_{F \text { max }}$, densities, $F_{\text {im }}$ has a negative slope, decreasing with increasing density. For intermediate densities, $\rho_{F \text { max }}>\rho>\rho_{F \text { max }}, F_{\text {im }}$ increases with density. The density, $\rho_{F \text { min }}^{*} \approx 0.1$, is almost temperature independent. The location of the maximum in $F_{\text {im }}(\rho)$ curve is $\rho_{F \max }^{*} \approx 0.4$ at low temperatures, but shifts to lower densities with increasing $T^{*}$.

A comparison of the behavior of $F_{\text {im }}(\rho)$, illustrated in Fig. 6, and $D(\rho)$, illustrated in Fig. 3 shows that the density of minimum $F_{\text {im }}$ coincides with the density of minimum $D$. In contrast, the density of maximum $F_{\text {im }}$ occurs at densities much higher than the density of maximum diffusivity. More- 

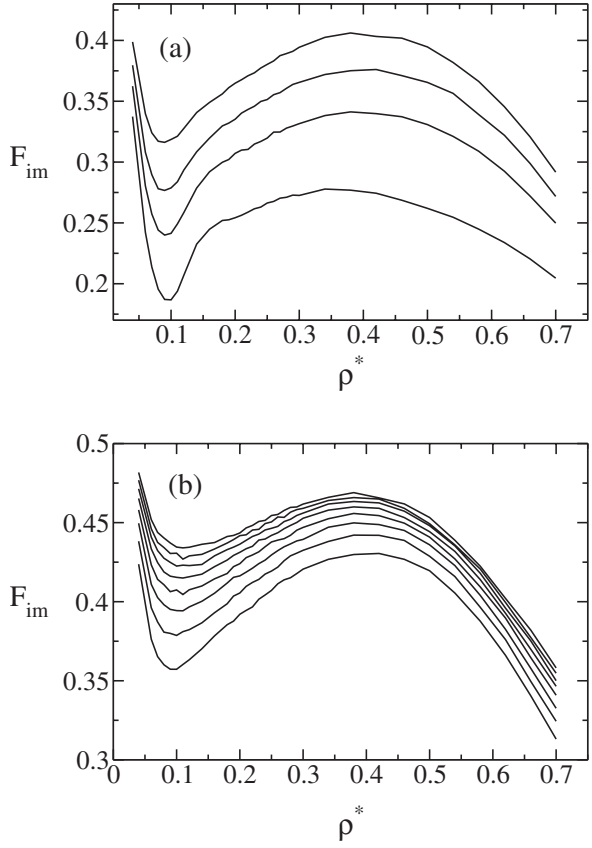

FIG. 6. (a) Fraction of imaginary modes against density for fixed temperatures $T^{*}=0.20,0.30,0.40,0.55$ from bottom to top. (b) Fraction of imaginary modes against density for fixed temperatures $T^{*}$ $=0.80,1.0,1.20,1.40,1.60 .1 .80,2.0,2.2$ from bottom to top.

over, the region in which $F_{\text {im }}$ shows an anomalous increase with compression persists to very high temperatures, well above the temperature for onset of structurally anomalous behavior. In order to understand this behavior, we compare the zeroth, first and second derivatives of the potential as a function of pair separation with $F_{\text {im }}$ for $T^{*}=1.0$ plotted as a function of the mean interparticle separation, $\rho^{-1 / 3}$ in Fig. 7. It is immediately obvious that the location of the minimum of $F_{\text {im }}$ coincides with the location of the minimum of the second derivative. For densities lower than this minimum, the second derivative increases and the number of imaginary modes decreases. Clearly, this effect persists in the hightemperature fluid where binary collisions dominate the dynamics since it reflects the curvature of the pair interaction.

\section{INM SPECTRA AND LIQUID-STATE ENTROPY}

In Sec. III, we discuss the possibility of partitioning the entropy of a liquid into contributions, $S_{r}$ and $S_{\mathrm{im}}$, associated with real and imaginary branches respectively of the INM

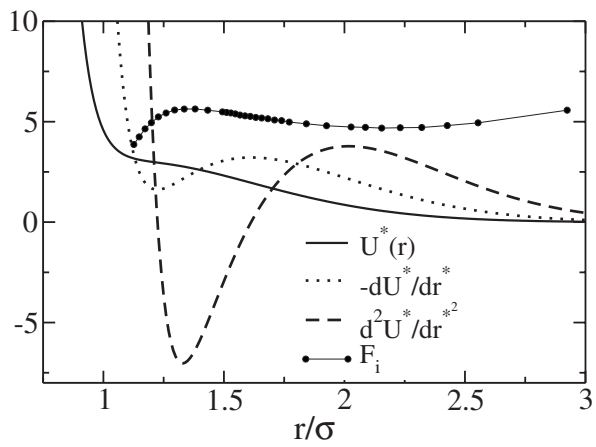

FIG. 7. Potential, force, and second derivative of the potential in units of $\epsilon$ and $F_{\mathrm{im}} * 15.0-1.0$ for $T^{*}=1.0$ vs reduced distance.

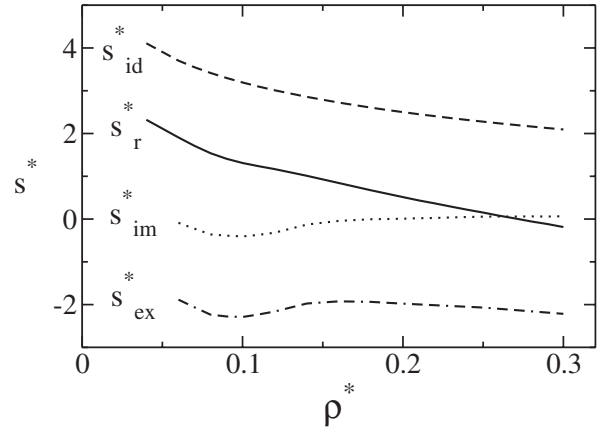

FIG. 8. $s_{\mathrm{id}}, s_{r}^{*}, s_{\mathrm{im}}^{*}$, and $s_{\mathrm{ex}}^{*}$ vs density for $T^{*}=0.2$.

spectrum. The $S_{r}$ contribution is directly derived from the frequency distribution of the real branch while the $S_{\text {im }}$ contribution is given by $S_{\mathrm{im}}=S-S_{r}$. In order to get a better understanding of the role played by each contribution to the entropy, Fig. 8 shows the behavior with density for a fixed temperature of $s_{\mathrm{im}}^{*}, s_{r}^{*}, s_{\mathrm{id}}^{*}$, and $s_{\mathrm{ex}}^{*}$. It is immediately evident that $s_{r}^{*}$ shows a very similar density dependence to $s_{\mathrm{id}}^{*}$, even though the numerical value of $s_{r}^{*}$ is significantly lower. Other than a small inflection at $\rho^{*} \approx 0.1$, the contribution of the real INM modes to the entropy carries virtually no signature of the structural, entropy or diffusivity anomalies. The nonmonotonic behavior of $s_{\mathrm{ex}}^{*}$ in the anomalous regime seems to be reflected only in $s_{\mathrm{im}}^{*}$. The strong resemblance between $s_{\mathrm{id}}^{*}$ and $s_{r}^{*}$ in $T$-and $\rho$-dependence suggests that this term reflects the generic effects of thermal fluctuations and spatial confinement on the entropy but in general it is insensitive to the structural details associated with the interplay between the two length scales in the anomalous regime.

Figure 9 compares, for various isotherms, the density dependence of three different entropy estimators: (a) $s_{\mathrm{im}}^{*}$, the imaginary mode contribution to the entropy, (in the graph, form the value of $s_{\mathrm{im}}^{*}$ it is subtracted 2), (b) $s_{2}^{*}$, the pair correlation entropy, and (c) $s_{\omega_{E}}^{*}$, the entropy estimated using the Einstein model for the liquid, Eq. (13). As discussed in Sec. IV A, the $s_{2}^{*}$ behavior is very similar in density and temperature dependence to $s_{\mathrm{ex}}^{*}$, indicating that the pair correlation contribution to the entropy is sufficient to capture the essential features of the anomalies. $s_{\omega_{E}}^{*}$, derived from the Einstein model, is very similar to $s_{r}^{*}$, presumably because of

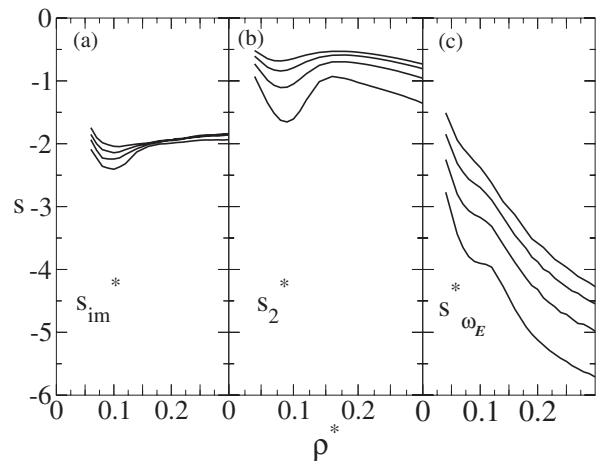

FIG. 9. (a) $s_{\text {im }}^{*}-2.0$ obtained from Eq. (16) with $s_{\text {im }}^{*}$ obtained from Ref. 35, (b) $s_{2}^{*}$ and (c) from the Einstein Frequency. See the text for more details. In all cases, the entropy is plotted against density for fixed temperatures $T^{*}=0.20,0.30,0.40$ and 0.50 from bottom to top. 


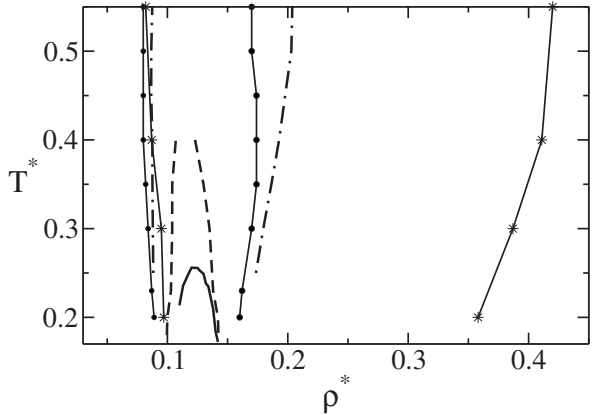

FIG. 10. Cascade of waterlike anomalies in the density-temperature plane. The solid line limits the region of density anomaly, the dashed line illustrates the region of diffusion anomaly and the dot-dashed line shows the region of structural anomaly. The filled circles represent the density of minimum and maximum $s_{2}$ and the stars represent the region of minimum and maximum $F_{\text {im }}$.

the use of the harmonic oscillator representation of the entropy. The nonmonotonic behavior of $\omega_{E}(\rho)$ results in small plateau at low temperatures. The overall decrease in $S_{\omega_{E}}$ with density is much too steep and monotonic compared with $s_{\mathrm{ex}}^{*}$ and $s_{2}^{*}$. In contrast, $s_{\text {im }}^{*}=s^{*}-s_{r}^{*}$, captures the behavior of the entropy within the anomalous region very successfully. The relative displacement of $s_{\text {im }}^{*}(\rho)$ curves for different isotherms is very small, consistent with the earlier observation that the overall effect of thermal and free volume effects is better captured by $s_{r}^{*}$.

As a summary of the insights obtained from INM analysis and pair correlation entropy into the nested cascade of anomalies picture of waterlike liquids, we use the extrema in $F_{\text {im }}(\rho)$ and $s_{2}^{*}(\rho)$ curves to define additional anomalous regimes within which $\left(\partial F_{\mathrm{im}} / \partial \rho\right)_{T}>0$ and $\left(\partial s_{2}^{*} / \partial \rho^{*}\right)_{T}>0$ respectively, as shown in Fig. 10. The low density boundaries of anomalous regime in $t, F_{\text {im }}$, and $s_{2}^{*}$ almost coincide, reflecting the onset of the steep repulsive wall. The highdensity boundary of the anomalous regime of $F_{\text {im }}$ occurs at very high densities. In contrast, the high density limit of the anomalous regime in $s_{2}^{*}$ is very close to that defined by $t$, and also by $s_{\text {im }}^{*}$ though the latter is not shown in the figure.

\section{CONCLUSIONS}

This paper explores the connection between entropy, diffusivity and the PEL of a core-softened fluid with waterlike anomalies using MD simulations and INM analysis.

We demonstrate that the diffusivity and the excess entropy of a core-softened fluid with isotropic pair interactions obey Rosenfeld-type excess entropy scaling of transport properties. The use of macroscopic reduction parameters for the diffusivity based on temperature and density is particularly appropriate for fluids with multiple length scales where defining an effective hard-sphere radius is inappropriate. We also show that the substituting the excess entropy by the pair correlation entropy leads to a weak isochore dependence of the Rosenfeld-scaling parameters, not seen in simple liquids but observed in other waterlike liquids. ${ }^{68}$

The INM spectra, including the Einstein frequency and the fraction of imaginary modes, is computed over a wide range of temperatures and densities. INM analysis is shown to provide unexpected insights into the dynamical consequences of the interplay between length scales characteristic of anomalous fluids that cannot be obtained from an equilibrium transport property such as the diffusivity.

Both the real and imaginary branches of the INM spectra exhibit bimodality that has so far not been observed. As a function of density along an isotherm, the bimodality in the real branch of the INM spectrum persists to very high densities well beyond the structurally anomalous region. In contrast, the bimodality of the imaginary branch is much more closely correlated with the region of the structural anomaly. The bimodal character of the both branches of the INM spectrum suggests that such core-softened fluids may show multiple time-scale behavior similar to that seen in hydrogenbonded systems.

The Einstein frequency shows an essentially monotonic dependence on density along an isotherm. The temperature dependence of the Einstein frequency is weak and monotonically decreasing with temperature at low densities and nonmonotonic at high temperatures. In contrast to the Einstein frequency, the fraction of imaginary frequencies shows very anomalous behavior in comparison to simple liquids, with an extended density regime over which $F_{\text {im }}$ increases with increasing density. While the low density boundary of this region coincides with that of the structural anomaly, the high density boundary occurs at very high densities well beyond the structurally anomalous regime. Previous INM studies of liquids have largely connected the diffusivity with the fraction of imaginary modes. Our results show that information about diffusivity is largely contained in the behavior of the imaginary branch of the INM spectra, but factors such as the bimodality of the frequency distribution in this branch must be considered in addition to $F_{\mathrm{im}}$.

Given the validity of excess entropy scaling for the diffusivities, we introduce INM-based estimators of the entropy, to connect the energy landscape with liquid state thermodynamics and kinetics. The conceptually simplest INM-based estimator is to treat the liquid as a collection of 3D harmonic oscillators vibrating at a single frequency, corresponding to the Einstein frequency. The Einstein model entropy shows a very steep decrease with density along isotherms with a very weak signature of the onset of the structurally anomalous regime.

An alternative approach to developing an INM-based estimator of the entropy that we have explored is to assume that the total entropy of the fluid can be written as a sum of contributions from $3 N F_{r}$ harmonic modes and $3 N F_{\text {im }}$ imaginary modes. The real branch of the INM spectrum can be used to estimate the harmonic contribution, $s_{r}^{*}$, exactly. The entropy contribution of the imaginary branch, $s_{\mathrm{im}}^{*}$, is then given by the difference of the thermodynamic entropy, $s$, and the real branch contribution, $s_{r}^{*}$. The temperature and density dependence of $s_{r}^{*}$ carries virtually no signature of the liquidstate anomalies, and seems to reflect only the generic effects of thermal fluctuations and spatial confinement on the entropy In contrast, $s_{\mathrm{im}}^{*}=s^{*}-s_{r}^{*}$, captures the behavior of the entropy within the anomalous region very successfully though the relative displacement of $s_{\mathrm{im}}^{*}(\rho)$ curves for different isotherms is too small. The extrema in $s_{\text {im }}^{*}$ define a region 
of anomalous entropy behavior in the density-temperature plane that is almost identical as the region within which $\left(\partial S_{2} / \partial \rho\right)_{T}>0$.

The overall and somewhat unexpected outcome of our INM analysis of a core-softened waterlike fluid is that the real and imaginary frequency branches show very different sensitivities to the dynamical consequences of the interplay between two length scales in the anomalous regime of the liquid. Moreover, the entropy contribution from the imaginary frequency modes of the INM spectrum reflects the anomalous behavior of the excess entropy and diffusivity characteristic of waterlike fluids, but the real frequency branch does not.

\section{ACKNOWLEDGMENTS}

This work is supported by the Indo-Brazil Cooperation Program in Science and Technology of the CNPq (Brazil) and DST (India).This work is also partially supported by the CNPq through the INCT-FCx. The authors would like to thank Murari Singh for help with preparing some of the figures.

${ }^{1}$ F. H. Stillinger and T. A. Weber, Phys. Rev. A 25, 978 (1982).

${ }^{2}$ P. G. Debenedetti and F. H. Stillinger, Nature (London) 410, 259 (2001).

${ }^{3}$ D. J. Wales, Energy Landscapes: With Applications to Clusters, Biomolecules and Glasses (Cambridge University Press, Cambridge, 2003).

${ }^{4}$ J.-P. Hansen and I. R. McDonald, Theory of Simple Liquids (Academic, London, 2002).

${ }^{5}$ F. Sciortino, E. La Nave, and P. Tartaglia, Phys. Rev. Lett. 91, 155701 (2003).

${ }^{6}$ I. Saika-Voivod, P. H. Poole, and F. Sciortino, Nature (London) 412, 514 (2001).

${ }^{7}$ G. Franzese, G. Malescio, A. Skibinsky, S. V. Buldyrev, and H. E. Stanley, Nature (London) 409, 692 (2001).

${ }^{8}$ A. Scala, F. W. Starr, and E. La Nave, F. Sciortino, and H. E. Stanley, Nature (London) 406, 6792 (2000).

${ }^{9}$ E. La Nave, A. Scala, F. W. Starr, H. E. Stanley, and F. Sciortino, Phys. Rev. Lett. 84, 4605 (2000).

${ }^{10}$ E. A. Jagla, Phys. Rev. E 58, 1478 (1998).

${ }^{11}$ H. M. Gibson and N. B. Wilding, Phys. Rev. E 73, 061507 (2006).

${ }^{12}$ L. Xu, P. Kumar, S. V. Buldyrev, S.-H. Chen, P. Poole, F. Sciortino, and H. E. Stanley, Proc. Natl. Acad. Sci. U.S.A. 102, 16558 (2005).

${ }^{13}$ A. Barros de Oliveira, M. C. Barbosa, and P. A. Netz, Physica A 386, 744 (2007).

${ }^{14}$ A. Barros de Oliveira, P. A. Netz, T. Colla, and M. C. Barbosa, J. Chem. Phys. 124, 084505 (2006).

${ }^{15}$ A. B. de Oliveira, P. A. Netz, T. Colla, and M. C. Barbosa, J. Chem. Phys. 125, 124503 (2006).

${ }^{16}$ D. Y. Fomin, D. Frenkel, N. V. Gribova, and V. N. Ryzhov, J. Chem. Phys. 129, 064512 (2008).

${ }^{17}$ S. A. Egorov, J. Chem. Phys. 128, 174503 (2008).

${ }^{18}$ J. Mittal, J. R. Errington, and T. M. Truskett, J. Chem. Phys. 125, 076102 (2006).

${ }^{19}$ J. R. Errington and P. G. Debenedetti, Nature (London) 409, 318 (2001).

${ }^{20}$ P. A. Netz, F. W. Starr, H. E. Stanley, and M. C. Barbosa, J. Chem. Phys. 115, 344 (2001)

${ }^{21}$ P. A. Netz, F. W. Starr, M. C. Barbosa, and H. E. Stanley, J. Mol. Liq 101, 159 (2002).

${ }^{22}$ S. Zhou, Phys. Rev. E 77, 041110 (2008).
${ }^{23}$ N. M. Barraz, E. Salcedo, and M. C. Barbosa, J. Chem. Phys. 131, 094504 (2009).

${ }^{24}$ P. Camp, Phys. Rev. E 68, 061506 (2003).

${ }^{25}$ Y. Rosenfeld, Phys. Rev. A 15, 2545 (1977).

${ }^{26}$ Y. Rosenfeld, Chem. Phys. Lett. 48, 467 (1977).

${ }^{27}$ Y. Rosenfeld J. Phys.: Condens. Matter 11, 5415 (1999).

${ }^{28}$ M. Dzugutov, Nature (London) 381, 137 (1996).

${ }^{29}$ J. J. Hoyt, M. Asta, and B. Sadigh, Phys. Rev. Lett. 85, 594 (2000).

${ }^{30}$ A. Samanta, S. M. Ali, and S. K. Ghosh, Phys. Rev. Lett. 87, 245901 (2001).

${ }^{31}$ A. Samanta, S. M. Ali, and S. K. Ghosh, J. Chem. Phys. 123, 084505 (2005).

${ }^{32}$ R. Sharma, S. N. Chakraborty, and C. Chakravarty, J. Chem. Phys. 125, 204501 (2006).

${ }^{33}$ M. Agarwal and C. Chakravarty, Phys. Rev. E 79, 030202 (2009).

${ }^{34}$ M. Agarwal, A. Ganguly, and C. Chakravarty, J. Chem. Phys. 113, 15284 (2009).

${ }^{35}$ J. R. Errington, T. M. Truskett, and J. Mittal, J. Chem. Phys. 125, 244502 (2006).

${ }^{36}$ T. Goel, C. N. Patra, T. Mukherjee, and C. Chakravarty, J. Chem. Phys. 129, 164904 (2008).

${ }^{37}$ H. S. Green, The Molecular Theory of Fluids (North-Holland, Amsterdam, 1952).

${ }^{38}$ R. E. Nettleton and H. S. Green, J. Chem. Phys. 29, 1365 (1958).

${ }^{39}$ H. J. Raveché, J. Chem. Phys. 55, 2242 (1971).

${ }^{40}$ D. C. Wallace, J. Chem. Phys. 87, 2282 (1987).

${ }^{41}$ A. Baranyai and D. J. Evans, Phys. Rev. A 40, 3817 (1989).

${ }^{42}$ R. M. Stratt and M. Maroncelli, J. Phys. Chem. 100, 12981 (1996).

${ }^{43}$ R. M. Stratt, Acc. Chem. Res. 28, 201 (1995).

${ }^{44}$ T. Keyes, J. Phys. Chem. A 101, 2921 (1997).

${ }^{45}$ P. Shah and C. Chakravarty, J. Chem. Phys. 115, 8784 (2001).

${ }^{46}$ P. Shah and C. Chakravarty, J. Chem. Phys. 116, 10825 (2002).

${ }^{47}$ P. Shah and C. Chakravarty, Phys. Rev. Lett. 88, 255501 (2002).

${ }^{48}$ M. Cho, G. R. Fleming, S. Saito, I. Ohmine, and R. M. Stratt, J. Chem. Phys. 100, 6672 (1994).

${ }^{49}$ M. C. C. Ribeiro and P. A. Madden, J. Chem. Phys. 106, 8616 (1997).

${ }^{50}$ W.-X. Li, T. Keyes, and F. Sciortino, J. Chem. Phys. 108, 252 (1998).

${ }^{51}$ A. Mudi, C. Chakravarty, and R. Ramaswamy, J. Chem. Phys. 122, 104507 (2005).

${ }^{52}$ H. Thurn and J. Ruska, J. Non-Cryst. Solids 22, 331 (1976).

${ }^{53}$ Periodic table of the elements, http://periodic.lanl.gov/default.htm, 2007.

${ }^{54}$ G. E. Sauer and L. B. Borst, Science 158, 1567 (1967).

${ }^{55}$ S. J. Kennedy and J. C. Wheeler, J. Chem. Phys. 78, 1523 (1983).

${ }^{56}$ T. Tsuchiya, J. Phys. Soc. Jpn. 60, 227 (1991).

${ }^{57}$ C. A. Angell, R. D. Bressel, M. Hemmatti, E. J. Sare, and J. C. Tucker, Phys. Chem. Chem. Phys. 2, 1559 (2000).

${ }^{58}$ M. S. Shell, P. G. Debenedetti, and A. Z. Panagiotopoulos, Phys. Rev. E 66, 011202 (2002).

${ }^{59}$ P. H. Poole, M. Hemmati, and C. A. Angell, Phys. Rev. Lett. 79, 2281 (1997).

${ }^{60}$ H. Tanaka, Phys. Rev. B 66, 064202 (2002).

${ }^{61}$ M. Agarwal, R. Sharma, and C. Chakravarty, J. Chem. Phys. 127, 164502 (2007).

${ }^{62}$ M. Agarwal and C. Chakravarty, J. Phys. Chem. B 111, 13294 (2007).

${ }^{63}$ S.-T. Lin, M. Blanco, and W. A. Goddard, J. Chem. Phys. 119, 11792 (2003).

${ }^{64}$ A. Mudi, C. Chakravarty, and R. Ramaswamy, J. Chem. Phys. 124, 069902E (2006).

${ }^{65}$ R. Sharma, C. Chakravarty, and E. Milotti, J. Phys. Chem. B 112, 9071 (2008).

${ }^{66}$ M. Agarwal, H. R. Kushwaha, and C. Chakravarty, J. Phys. Chem. B 114, 651 (2010).

${ }^{67}$ G. Seeley and T. Keyes, J. Chem. Phys. 91, 5581 (1989).

${ }^{68}$ M. Agarwal, M. Singh, R. Sharma, M. P. Alam, and C. Chakravarty, J. Chem. Phys. B 114, 6995 (2010); arXiv:1004.3091. 